\title{
Enhanced Activity of the Free Radical Producing Enzyme Xanthine Oxidase in Hypoxic Rat Liver \\ Regulation and Pathophysiologic Significance
}

Clifford A. Brass, Julianne Narciso, and John L. Gollan

Gastroenterology Division, Brigham and Women's Hospital, Department of Medicine, Harvard Medical School, and the Harvard Digestive Diseases Center, Boston, Massachusetts 02115

\begin{abstract}
It has been widely proposed that conversion of xanthine dehydrogenase (XDH) to its free radical-producing form, xanthine oxidase (XOD), underlies ischemic/reperfusion injury, although the relationship of this conversion to hypoxia and its physiologic control have not been defined. This study details the time course and control of this enzymatic interconversion. In a functionally intact, isolated perfused rat liver model, mean $\%$ XOD activity increased as a function of both the duration ( 25 to $45 \%$ in $3 \mathrm{~h})$ and degree $(r=0.97)$ of hypoxia. This process was markedly accelerated in ischemic liver by an overnight fast ( 45 vs. $30 \%$ at $2 \mathrm{~h}$ ), and by imposing a short period of in vivo ischemia (cardiopulmonary arrest $72 \%$ ). Moreover, only under these conditions was there a significant rise in the XOD activity due to the conformationally altered XDH molecule (XODc, $18 \%$ ), as well as concomitant morphologic injury. Neither circulating white blood cells nor thrombosis appeared to contribute to the effects of in vivo ischemia on enzyme conversion. Thus, it is apparent that conversion to the free radical-producing state, with high levels of XOD activity and concurrent cellular injury, can be achieved during a relatively short period of hypoxia under certain well-defined physiologic conditions, in a time course consistent with its purported role in modulating reperfusion injury. These data also suggest that the premorbid condition of organ donors (e.g., nutritional status and relative state of hypoxia) is important in achieving optimal organ preservation. (J. Clin. Invest. 1991. 87:424-431.) Key words: xanthine oxidase $\cdot$ reperfusion injury $\bullet$ hypoxia $\bullet$ liver injury • ischemia
\end{abstract}

\section{Introduction}

As the importance of understanding the mechanism(s) of tissue injury associated with ischemia and reperfusion has increased with the application of new therapeutic strategies (e.g., liver transplantation, cardiac reperfusion), research in this field has focused on free radical-mediated injury. Based on the observations of Granger and colleagues in the intestine $(1,2)$, and

Presented in part at the American Association for the Study of Liver Diseases Meeting, Chicago, October 1988.

Address reprint requests to Dr. Brass, University of Pennsylvania School of Medicine, Clinical Research Building 600, 422 Curie Boulevard, Philadelphia, PA 19104-6144.

Received for publication 22 February 1990 and in revised form 25 July 1990

J. Clin. Invest.

(c) The American Society for Clinical Investigation, Inc. $0021-9738 / 91 / 02 / 0424 / 08 \quad \$ 2.00$

Volume 87, February 1991, 424-431 similar studies on the heart $(3,4)$ and kidney $(5)$, the concept of "reperfusion injury" emerged. This hypothesis proposes that there is a distinct injury associated with the production of toxic free radical species after the resupply of blood (or oxygen) to previously ischemic (or hypoxic) tissues. Indeed, in many circumstances reperfusion injury may be more important than the initial hypoxic insult.

It has been proposed that the activity of the free radical-producing enzyme xanthine oxidase (XOD), ${ }^{1}$ which is formed from alteration of xanthine dehydrogenase (XDH), increases during ischemia, and that the reduced substrates xanthine and hypoxanthine accumulate from the breakdown of ATP (6). With the reintroduction of oxygen, which is the limiting substrate in this setting, a burst of free radical production and cell injury ensues $(7,8)$. This hypothesis has formed the major conceptual basis for most of the recent research on organ preservation in the liver, heart, and kidney.

Despite widespread acceptance, there is little direct experimental evidence to support this concept. In fact, much of the data questions the existence of reperfusion injury in the liver (9, 10, Lipsky, M., and J. L. Gollan, unpublished data), and many of those observations in support of the reperfusion hypothesis are indirect and depend on the use of inhibitors of XOD activity (allopurinol) or free radical scavengers (e.g., superoxide dismutase, catalase; 11-14), which may have additional effects. Few investigators have actually documented $\mathrm{XDH}$ and XOD conversion in hypoxic tissues, although recent in vitro studies in excised liver $(15,16)$ and gut $(17)$ suggest a slow time course for this process inconsistent with its purported role in mediating reperfusion injury. Recently, it has been proposed that circulating white blood cells may be important in amplifying ischemic and reperfusion injury in the heart $(4,18$, 19), gut (20), and liver (21). Despite this shift in focus to endothelial cell and neutrophil interactions that may decrease tissue perfusion (i.e., the no-reflow phenomenon; 22), the control of XOD activity remains a key issue, because this theory also postulates the production of free radical species via xanthine oxidase (or some other as yet undefined mechanism).

In light of the contradictory data concerning the relevance of reperfusion injury, we proceeded to systematically examine the basis of this theory, namely the conversion of XDH to XOD during hypoxia. Specifically, the relationship of this process to hypoxia, and its occurrence and modulation in a physiologically intact organ have not been defined. Our findings characterize the conversion of XDH to XOD activity in a physiologically viable system (i.e., isolated perfused liver), and its

1. Abbreviations used in this paper: CPA, cardiopulmonary arrest; DTT, dithiothreitol; IPRL, isolated perfused rat liver; wbc, white blood cells; XDH, xanthine dehydrogenase; XOD, xanthine oxidase. 
relation to the duration and severity of hypoxia. We also have investigated the factors that regulate this process in anoxic liver, and thereby accelerate the temporal course of the conversion into a time frame consistent with its postulated role in reperfusion injury. Collectively, these data advance our understanding of the classic theory of reperfusion injury and clarify the potential role of XOD vis-a-vis more recent paradigms involving endothelial cell/neutrophil interactions.

\section{Methods}

Isolated perfused rat liver. Adult Sprague-Dawley rats weighing 250$350 \mathrm{~g}$, either fed ad libitum (fed) or deprived of food for $16 \mathrm{~h}$ (starved), were used as liver donors. Each rat was anesthetized by the intraperitoneal administration of sodium pentobarbital $(50 \mathrm{mg} / \mathrm{kg}$; Abbott Laboratories, Chicago, IL), the abdomen was opened and the bile duct cannulated with PE-10 tubing. The portal vein was then cannulated with a 16-gauge catheter, flushed with 400 IU heparin (Elkin-Sinn Inc., Cherry Hill, NJ) in $0.8 \mathrm{ml}$ of $0.15 \mathrm{M} \mathrm{NaCl}$, and perfusion initiated in situ with an oxygenated modified Krebs-Ringer bicarbonate buffer (23).

The recirculating perfusion system, containing $50 \mathrm{ml}$ of perfusate, has previously been described in detail (23). The medium was passed through a membrane oxygenator consisting of $4 \mathrm{~m}$ of silastic tubing $(0.147 \mathrm{~cm}$ i.d., Dow-Corning Corp., Midland, $\mathrm{MI})$, coiled in a closed container into which $95 \% \mathrm{O}_{2} / 5 \% \mathrm{CO}_{2}$ or $95 \% \mathrm{~N}_{2} / 5 \% \mathrm{CO}_{2}$ flowed at a rate of $\sim 400 \mathrm{ml} / \mathrm{min}$. The perfusate was passed sequentially through a temperature-sensitive probe (coupled to a thermostat set at $37^{\circ} \mathrm{C}$ ), a stainless steel filter screen (Millipore Corp., Bedford, MA), a bubble trap and a pressure gauge, before entering the portal vein via the Teflon catheter.

Isolated livers were perfused with a hemoglobin-free, Krebs-Ringer bicarbonate medium (23), or with a perfluorochemical emulsion (Oxypherol, Alpha Therapeutics Corp., Los Angeles, CA) to enhance oxygen-carrying capacity. Oxygen delivery to the liver was adjusted by changing the perfusate and/or the exchange gas. The perfusate was sampled immediately before inflow into the portal vein catheter, and as it emerged from the liver through the IVC cannula. Perfusion rates were $2.3 \mathrm{ml} / \mathrm{min}$ per $\mathrm{g}$ liver and $1.7 \mathrm{ml} / \mathrm{min}$ per $\mathrm{g}$ for the Krebs and Oxypherol media, respectively. Perfusate oxygen saturation was measured using a biological oxygen monitor (model 5300, Yellow Springs Instrument Inc., Yellow Springs, $\mathrm{OH}$ ) set in the batch mode at $37^{\circ} \mathrm{C}$. Hepatic oxygen consumption was calculated on the basis of partial oxygen pressure in the portal and hepatic veins, the perfusion flow rate, the liver weight, and the oxygen carrying capacity of the perfusate (24). Bile was collected into tared tubes throughout each experiment.

Experimental models. In the experiments with global hepatic ischemia (anoxia), the operative technique was as outlined above, except that the bile duct was not cannulated, and the liver was placed in a beaker of unstirred $\mathrm{Krebs}$ medium in a $37^{\circ} \mathrm{C}$ water bath. To induce neutropenia, adult rats were exposed to 1,000 rads of whole-body irradiation (Gamma Cell 40, Atomic Energy Canada, Ltd., Kanata, Canada) at a rate of $88 \mathrm{rads} / \mathrm{min}, 10$ days before study. This procedure reduced circulating white blood cells from 5,000 to $10,000 \mathrm{wbc} / \mathrm{ml}$ to $<500 \mathrm{wbc} / \mathrm{ml}$ within $10 \mathrm{~d}$.

The "cardiopulmonary arrest" (CPA) model of in vivo ischemia was prepared by making a large incision in the diaphragm of an anesthetized rat and waiting $10 \mathrm{~min}$ before proceeding with the surgical procedure. This intervention created a period of in vivo hepatic ischemia, because complete cessation of respiratory movement and effective cardiac contraction occurred during this period. Preheparinization of animals was achieved, as required, by injection of $500 \mathrm{IU}$ of sodium heparin into the inferior vena cava of the anesthetized rat $4 \mathrm{~min}$ before initiation of the CPA procedure.

At the conclusion of each experiment the liver was blotted dry and weighed. The degree of liver edema was calculated as the difference in liver weight before and after each experiment divided by the preexperi- mental liver weight. The latter was estimated from accumulated scaling data (Gollan, J. L., unpublished data), based on the rat body weight $(0.032 \mathrm{~g} / \mathrm{kg}$ body wt starved, and $0.0375 \mathrm{~g} / \mathrm{kg}$ body wt fed). A small wedge was resected from the right lobe of the liver at the termination of each experiment, placed in 10\% formalin in saline, sectioned, and stained with hematoxylin and eosin. Where indicated cell death was histologically quantitated by a modification ( $7 \mathrm{~min}$ Trypan blue perfusion, 2 min fixation, no rinse period) of the Trypan blue perfusion technique (25).

Xanthine oxidase/xanthine dehydrogenase assays. On completion of each experiment the liver was rapidly frozen in liquid nitrogen, ground with a mortar and pestle, and homogenized in a Polytron Homogenizer (Brinkman Instruments, Westbury, NY) for $45 \mathrm{~s}$, after the addition of 4 vol of a buffered solution ( $\mathrm{pH} 7.8$ ) containing $0.05 \mathrm{M}$ potassium phosphate, $0.1 \mathrm{M}$ EDTA, $0.2 \mathrm{M}$ phenylmethyl-sulfonyl fluoride (to inhibit irreversible XDH-to-XOD conversion) and $0.5 \mathrm{mM}$ dithiothreitol (to inhibit reversible XDH-to-XOD conversion). In those experiments where conformationally altered xanthine oxidase activity (XODc) was measured, one-half of the frozen liver sample (by weight) was homogenized in the phosphate buffer without dithiothreitol (DTT). XODc activity was calculated as the difference in XOD activity measured in homogenate from the same liver, with (XODe) and without (XODe + XODc) DTT. There was no difference in total oxidase plus dehydrogenase activity when DTT was omitted from the homogenate.

Homogenate was centrifuged at $27,000 \mathrm{~g}$ for $45 \mathrm{~min}$, and the supernatant passed through a Sephadex G-25 column (Pharmacia Fine Chemicals, Uppsala, Sweden) to remove any endogenous inhibitors. The eluate was assayed immediately for XDH and XOD activity, using a modification of the method of Waud and Rajugopalan (26). Uric acid formation was measured at $295 \mathrm{~nm}$ in the presence (XOD + XDH) or absence (XOD) of $6 \mathrm{mM} \mathrm{NAD}^{+}$at $37^{\circ} \mathrm{C}$. All assays were performed in triplicate. Xanthine dehydrogenase activity was calculated as the difference between these two values, and \% XOD was expressed as XOD activity divided by the sum of the XOD and XDH activities. There were no significant differences in total XOD and XDH activity between any of the experimental groups, so that changes in \% XOD activity reflected the conversion from the dehydrogenase to the oxidase form of the enzyme. Total xanthine oxidase plus dehydrogenase activity was $10.78 \pm 0.45 \mu \mathrm{mol}$ uric acid formed/min per $\mathrm{mg}$ protein (mean $\pm \mathrm{SE}$ ). Aspartate aminotransferase and lactate dehydrogenase activities in the perfusate were measured spectrophotometrically using commercial kits (Sigma Chemical Co., St. Louis, MO). Values $<100$ and $5,000 \mathrm{U} / \mathrm{ml}$ per $\mathrm{g}$ liver, respectively, reflect minimal injury in the isolated perfused rat liver (IPRL) system used (Brass, C. A., and J. L. Gollan, unpublished observation).

Statistical methods. The data are presented as mean and standard error. The unpaired Student's $t$ test was used to estimate the signifcance of the differences between mean values.

\section{Results}

\section{Studies with isolated perfused rat liver}

Conversion to xanthine oxidase activity during hypoxia. To determine whether conversion from XDH to XOD activity occurs in a functional model during hypoxia, experiments were performed using a recirculating IPRL system. The liver from fed rats was perfused with oxygenated Krebs-bicarbonate buffer for a 30-min equilibration period, before the initiation of hypoxic perfusion for a period of $180 \mathrm{~min}$. As shown in Fig. 1, $\%$ XOD activity increased from 25 to $45 \%$ during this period, with a close correlation $(n=7 ; r=0.97)$ between the duration of hypoxia and XOD activity.

Relationship between degree of hypoxia and xanthine oxidase conversion. To determine whether enzyme conversion was related to the level of hypoxia, XOD and XDH activities 


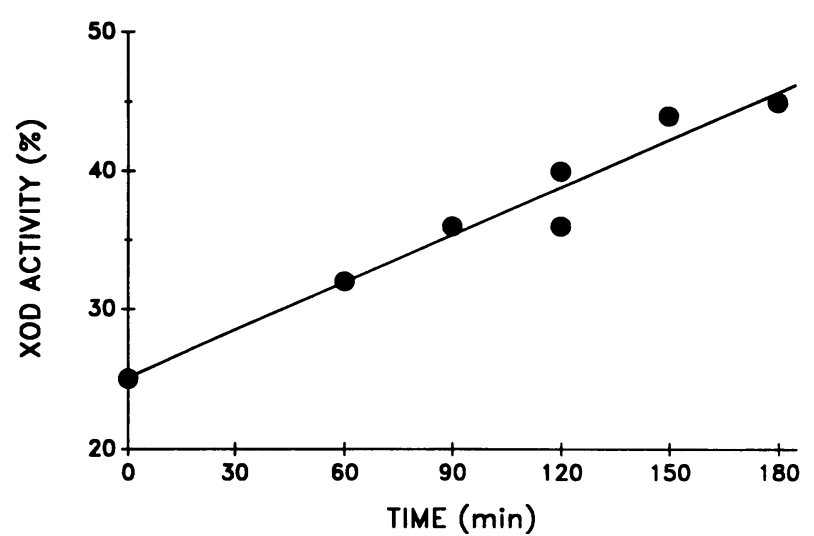

Figure 1. Conversion to xanthine oxidase activity (\% XOD) during hypoxia in IPRL. Liver from rats fed ad libitum was perfused with an oxygenated Krebs-bicarbonate buffer for $30 \mathrm{~min}$ before hypoxic perfusion for a defined period. Each point represents the \% XOD activity $[\mathrm{XOD} /(\mathrm{XOD}+\mathrm{XDH})]$ measured at the end of the perfusion period $(r=0.97)$.

were measured in the liver from fed animals exposed to different levels of oxygenation (Fig. 2). The level of oxygen consumption was inversely related to the $\%$ XOD activity determined at $2.5 \mathrm{~h}(n=24 ; r=0.92)$.

Hepatic function and viability. Because conversion of XDH to XOD has not been reported previously in a functionally intact system, the viability of the hypoxic livers was assessed using several different approaches. As shown in Fig. 3, hepatic oxygen consumption returned to near normal levels $\left(2.50 \pm 0.25 \mu \mathrm{mol} \mathrm{O}_{2} / \mathrm{min}\right.$ per $\left.\mathrm{g} ; 24\right)$ when oxygen was reintroduced after $2 \mathrm{~h}$ of hypoxic perfusion. Bile flow which decreases $40-60 \%$ due to bile salt depletion under fully oxygenated conditions (26), returned to comparable levels $(39 \pm 12 \%)$ after reoxygenation (Fig. 3). In addition, hepatocellular injury, as assessed by both AST and $\mathrm{LDH}$ release into the perfusate (18 \pm 1 and $1,007 \pm 256 \mu \mathrm{m} / \mathrm{ml}$ per $\mathrm{g}$ liver, respectively), as well as cell death measured by Trypan blue uptake (3\%) were ex-

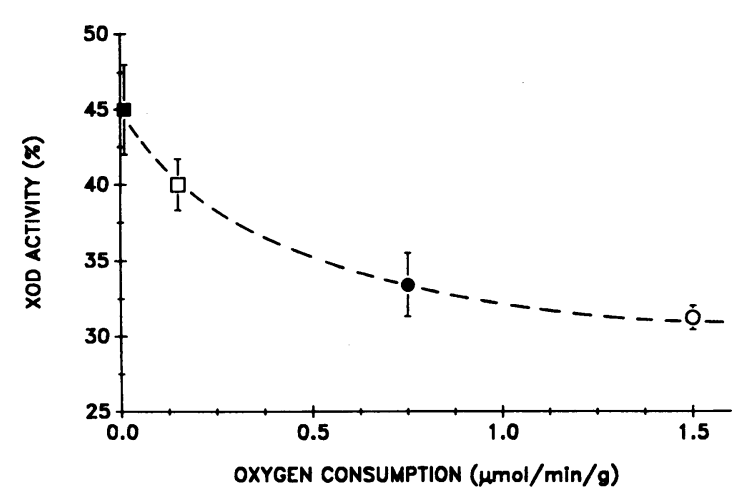

Figure 2. Conversion to xanthine oxidase activity as a function of hepatic oxygen consumption. Livers from fed rats were exposed to different levels of oxygenation by perfusion with oxygenated Oxypherol (o), oxygenated Krebs-bicarbonate buffer ( $\bullet$ ), nitrogensaturated Krebs buffer ( $\square$ ), or incubation in Krebs buffer ( $\square$ ) without perfusion for $150 \mathrm{~min}$. Oxygen consumption was calculated from the mean difference in $\mathrm{O}_{2}$ saturation measured in the portal and hepatic veins. Each point represents the mean \pm SE of the \% XOD conversion in six or more perfused livers $(r=0.92)$.

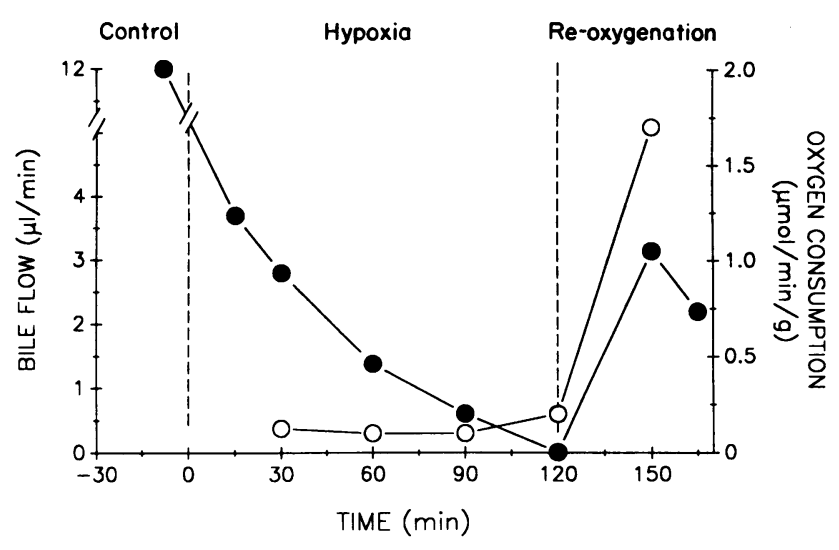

Figure 3. Hepatic oxygen consumption and bile flow during hypoxia and reoxygenation in the IPRL. Oxygen consumption $(0-0)$ and bile flow ( - $\bullet$ ) were measured in the liver of fed rats (representative experiment shown). The liver was perfused with nitrogen-saturated Krebs-bicarbonate buffer for $120 \mathrm{~min}$, followed by oxygenated Krebs buffer for $30 \mathrm{~min}$. The control period denotes the time of bile collection during the surgical procedure and before placement into the perfusion apparatus.

tremely low. Thus, based on these parameters, it is apparent that conversion to the XOD enzyme form during hypoxia occurs in functionally intact liver.

\section{Factors influencing xanthine dehydrogenase to oxidase conversion}

Effects of fasting. To further define the factors modulating the conversion of hepatic XDH to XOD, a model of global ischemia was employed. Consistent with previous observations $(15,16)$, there was a gradual increase in \% XOD activity in the liver of fed animals over the 2.5 -h period of anoxia. However, conversion was accelerated in the livers from fasted animals (Fig. 4); thus, after $2 \mathrm{~h}$ of anoxia, the $\%$ XOD was $50 \%$ higher in the livers of fasted than fed rats $(45 \pm 15 \%$ vs. $30 \pm 8 \%, P<0.01)$.

Cardiopulmonary arrest model. To simulate a period of in vivo ischemia, a 10-min period of cardiopulmonary arrest (CPA) was employed before excision of the rat liver. The slow conversion to XOD activity over a 2 -h period of anoxia in the livers from fed control animals $(n=16)$ was unchanged by the prior imposition of cardiopulmonary arrest $(n=11)$. However, after fasting for $16 \mathrm{~h}$, there was a marked increase in hepatic XOD activity in the animals subjected to cardiopulmonary arrest $(n=32)$, relative to the fasted controls $(n=17$; Fig. 4$)$. This difference was evident within $30 \mathrm{~min}$, and increased progressively at $60 \mathrm{~min}(47 \pm 4$ vs. $32 \pm 3 \%)$ and $90 \mathrm{~min}(57 \pm 9$ vs. $37 \pm 4 \%$ ). Hence, cardiopulmonary arrest had an effect on enzyme conversion only in the fasted animals. After $90 \mathrm{~min}$ of anoxia there was a twofold increase in \% XOD activity $(57 \pm 9 \%)$, compared with that observed in fed animals subjected to cardiopulmonary arrest $(29 \pm 4 \%$; Fig. 4). Similarly, starvation and cardiopulmonary arrest affected conversion to XOD only after a period of hypoxia, because the \% XOD activity before the onset of anoxia (at $0 \mathrm{~min}$ ) was comparable in all four groups.

Liver morphology. The imposition of cardiopulmonary arrest in fasted animals resulted in significantly greater $(P$ $<0.05$ ) hepatic edema relative to the anoxic livers from other 


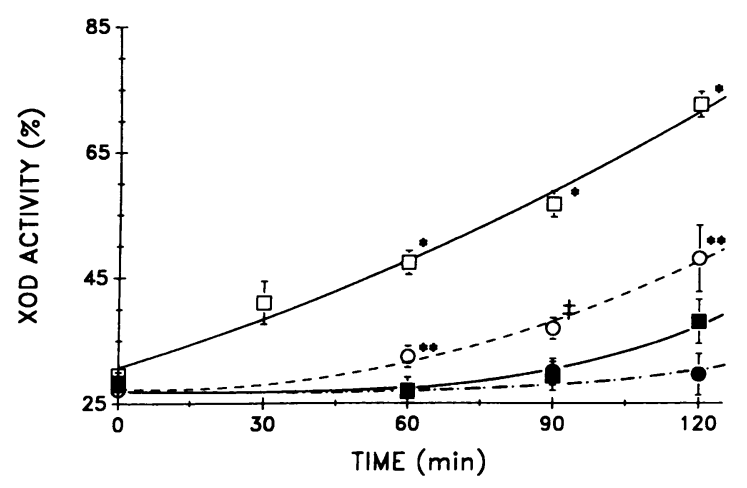

Figure 4. Conversion of xanthine dehydrogenase to XOD activity during ischemia. Isolated rat livers were placed in an unstirred Krebs-bicarbonate bath at $37^{\circ} \mathrm{C}$, and assayed for XOD activity, and XDH plus XOD activities after different periods of anoxia. Xanthine oxidase activity (\% XOD) is shown as percent total oxidase plus dehydrogenase activity $(\mathrm{XOD}+\mathrm{XDH})$ as a function of the duration of anoxia. Each point represents the mean $\pm \mathrm{SE}$ of at least three rat livers $(n=76)$. Livers were obtained from rats fed ad libitum $(\bullet-\bullet \bullet)$ or fasted for $16 \mathrm{~h}(0--0)$. Cardiopulmonary arrest (-) involved cutting the diaphragm of the anesthetized rat and delaying completion of surgical removal of the liver for a 10-min period of in vivo ischemia. The effect of this intervention in fasted animals is denoted by $(\square-\square) .{ }^{*} P<0.01$ vs. starved or fed/ cardiopulmonary arrest; ${ }^{* *} P<0.01$ vs. fed; ${ }^{\ddagger} P<0.05$ vs. fed.

groups. The increase in water content in the liver after anoxia in the starved/CPA group $(51 \pm 4 \%)$ was almost twice that in the livers of fed $(24 \pm 3 \%)$, fed/CPA $(29 \pm 4 \%)$, or starved $(20 \pm 4 \%)$ animals. This was accompanied by marked histologic changes. Whereas global ischemia for $90 \mathrm{~min}$ in the liver from fasted rats caused no discernible alteration in histologic appearance of hematoxylin and eosin stained liver, the same period of anoxia caused marked cellular injury in the livers from the starved/ CPA group (Fig. 5). In particular, extensive vacuolization of hepatocytes, reflective of loss of cellular volume control, was noted, as well as eosinophilic degeneration indicative of hepatocellular death. No zonal differences in the pattern of injury were noted.

Determination of DTT-labile XOD activity. Because dithiothreitol was present in the homogenization buffer, the data presented thus far reflect only the XOD activity present as the $\mathrm{XDH}$ cleavage product (XODe). However, it has been postulated (16) that XODc (DTT-labile XOD) may be the major intermediate for cleavage to XODe.

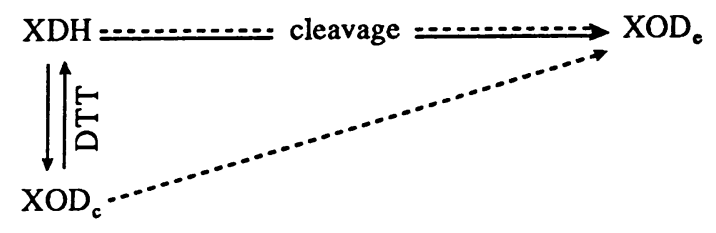

Total XOD activity, and hence the potential for free radical formation via this enzyme, is represented by the sum of XODC and XODe. To assess the potential importance of XODc, under the various hypoxic conditions imposed, activity of this form of the enzyme was also measured. As shown in Table I, DTT-labile xanthine oxidase activity (XODc) increased after $90 \mathrm{~min}$ of ischemia (anoxia) only in the liver of starved ani- mals, subjected to prior cardiopulmonary arrest. Before the onset of ischemia, no DTT-labile XOD activity was measurable in the liver. However, this activity increased progressively with time in the ischemic livers from the starved/CPA group, achieving a maximum of $18 \pm 2 \%$ at 90 min (Fig. 6). Thus, the sum of XODc and XODe activities, demonstrated a substantial shift toward the free radical-producing state during ischemia in the starved/CPA group. Three-quarters of the total $\mathrm{XDH}+\mathrm{XOD}$ activity was in the XOD form after $90 \mathrm{~min}$ of ischemia (Table I), increasing to $90 \%$ after $2 \mathrm{~h}$. Thus, conversion to XOD activity is virtually complete in the liver of the starved/CPA animals after a relatively short period of warm anoxia.

\section{Potential mechanisms of the "cardiopulmonary arrest" effect}

Role of thrombosis. To investigate the possibility that vascular thrombosis during the period of cardiopulmonary arrest was, at least in part, responsible for the effects of this maneuver, a group of animals was heparinized before the onset of cardiopulmonary arrest and subsequent hepatic resection. Heparinization of animals had no effect on the rate of formation of XOD activity $(70 \pm 4 \%, n=6)$ in the liver of starved/CPA rats, compared to nonheparinized animals $(73 \pm 4 \%, n=6)$, after $2.5 \mathrm{~h}$ of ischemia.

Role of circulating white blood cells. To investigate the possible relevance of circulating white blood cells as mediators of the increased XOD formation and histologic changes that followed in vivo ischemia (cardiopulmonary arrest) and subsequent ischemia, neutropenic animals were studied. As shown in Fig. 7, the imposition of cardiopulmonary arrest on starved neutropenic rats, followed by a 90 -min period of global anoxia, led to a significant increase in \% XOD activity (52 $5 \%$ ) compared with fed controls $(29 \pm 2 \%)$. No significant difference was noted with the similarly treated nonneutropenic animals $(57 \pm 2 \%)$. Thus, in this ischemic liver model, circulating white blood cells

Table I. Percent Conversion to Reversible (XODc) and Irreversible (XODe) Xanthine Oxidase Activity during Anoxia

\begin{tabular}{lccc}
\hline \multicolumn{1}{c}{ Procedure } & XODc & XODe & XODc $+\mathrm{e}$ \\
\hline Fed & $0 \pm 1$ & $30 \pm 2$ & 30 \\
Fed/CPA & $2 \pm 1$ & $30 \pm 2$ & 32 \\
Starved & $1 \pm 1$ & $37 \pm 3$ & 38 \\
Starved/CPA & $18 \pm 2^{*}$ & $57 \pm 2^{*}$ & 75 \\
\hline
\end{tabular}

Excised livers from rats fed ad libitum (fed) or fasted for $16 \mathrm{~h}$ (starved) were exposed to $90 \mathrm{~min}$ of ischemia in Krebs medium at $37^{\circ} \mathrm{C}$. Cardiopulmonary arrest (CPA) was imposed before the ischemic period, as detailed in Methods. Xanthine oxidase (\%) is expressed as the oxidase activity divided by the sum of xanthine oxidase and dehydrogenase activities. Irreversible, or enzymatically altered xanthine oxidase activity (XODe; see Methods) was assayed in the presence of the sulfhydryl protectant dithiothreitol. Reversible, or conformationally altered xanthine oxidase activity (XODc) was calculated as the difference between total xanthine oxidase activity (XODc + e; measured in the absence of dithiothreitol) and XODe. Data for XODc and XODe are shown as the mean \pm SE for four or more animals in each group $(n=52)$. Righthand column represents the sum of the mean XODc and XODe activities. ${ }^{*} P<0.01$. 

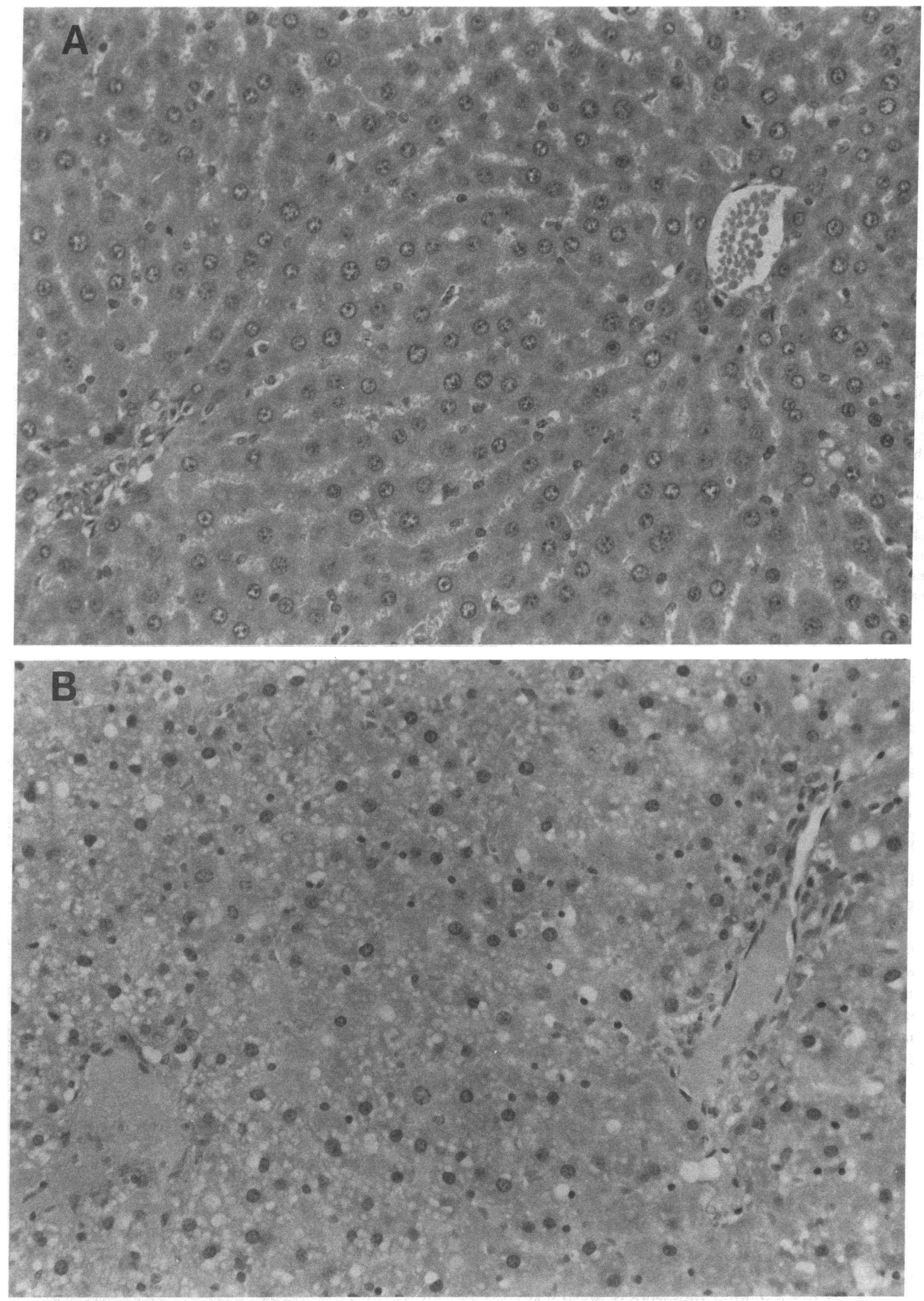

Figure 5. Morphologic appearance of hypoxic injury in the liver of animals fasted for $16 \mathrm{~h}$, with and without the imposition of a 10 -min period of cardiopulmonary arrest. Each liver was subjected to a 90 -min period of ischemia (Fig. 4), before fixation of a thin section of the lateral right lobe in $10 \%$ formalin and staining with hematoxylin and eosin. $(A)$ The liver (no cardiopulmonary arrest) appears well preserved, in contrast to the marked cellular damage noted when cardiopulmonary arrest preceded the hypoxic period $(B)$. 


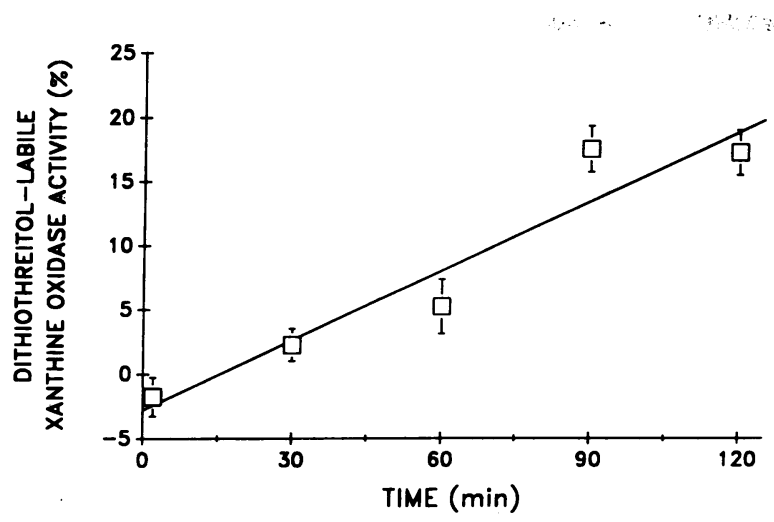

Figure 6. Conversion to reversible xanthine oxidase activity (XODc) during ischemia in the starved/cardiopulmonary arrest model. Livers excised from rats fasted for $16 \mathrm{~h}$ and subjected to a 10-min period of cardiopulmonary arrest (CPA; see Methods), were placed in unstirred Krebs-bicarbonate medium for up to $120 \mathrm{~min}$. At the end of the anoxic period, each liver was divided equally and assayed for xanthine oxidase and dehydrogenase activities in the presence or absence of the sulfhydryl protectant dithiothreitol. Xanthine oxidase activity (\%) was calculated as the oxidase activity divided by the total oxidase and dehydrogenase activities. Reversible or dithiothreitol labile xanthine oxidase activity (XODc) was calculated as the difference between \% XOD measured in the absence (XODc $+\mathrm{XODe}$ ) and presence (XODe) of dithiothreitol. Each point represents the mean $\pm \mathrm{SE}$ of least four livers.

do not appear to modulate the cardiopulmonary arrest-induced increase in XOD activity.

\section{Discussion}

These findings demonstrate that conversion from xanthine dehydrogenase to xanthine oxidase activity occurs during hypoxia in the IPRL (Fig. 1). Although this conversion process proceeds slowly, with an increase from 25 to $45 \%$ XOD activity over a 3-h period, the enzymatic transformation occurs in a

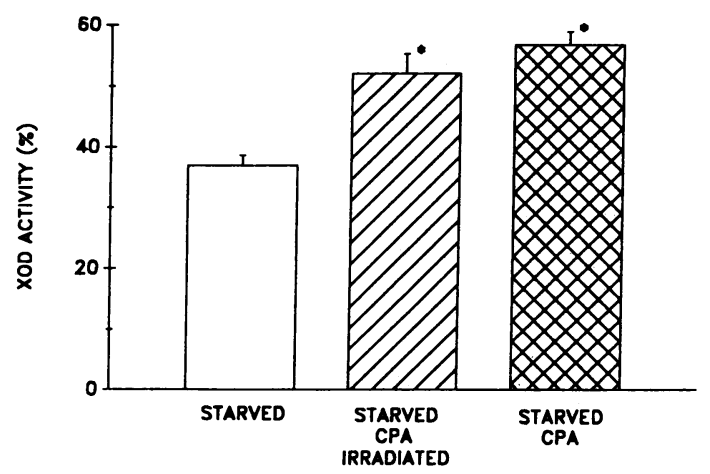

Figure 7. Effect of neutropenia on conversion to xanthine oxidase activity during anoxia. The livers from rats fasted for $16 \mathrm{~h}$ were placed in unstirred Krebs-bicarbonate buffer for $90 \mathrm{~min}$, and then assayed for XOD and XOD plus dehydrogenase activities. The cardiopulmonary arrest (CPA) procedure is described in Methods. Neutropenia $(<500 \mathrm{wbc} / \mathrm{ml})$ was achieved by whole-body irradiation ( 1,000 rads) $10 \mathrm{~d}$ before the experiment. Xanthine oxidase activity (\% XOD) is plotted as the percent of total oxidase plus dehydrogenase activities. The mean $\pm S E$ for at least seven livers per group are shown. ${ }^{*} P<0.01$ compared to starved group. relatively intact (low AST and LDH release, and low Trypan blue staining), functional liver, as reflected by its continued ability to utilize oxygen and to produce bile after reoxygenation. Moreover, the extent of enzyme conversion is related directly to the level of hypoxia (Fig. 2). These findings have been previously assumed by those investigators subscribing to the xanthine oxidase theory of reperfusion injury $(6,8)$. However, these data are the first to document conversion to the free radical-producing state (increased XOD activity) as a function of both the extent and duration of hypoxia in a functional organ.

These observations appear to support the dominant role of xanthine oxidase in mediating reperfusion injury in the liver, but they do not conclusively establish this paradigm for two reasons. First, no causal relationship between increased xanthine oxidase levels and cellular injury has been established. Second, the modest rise observed in XOD activity, raises the question of the physiologic relevance of this enzymatic conversion. To address the latter concern we investigated the variables that modulate and potentially enhance this response to hypoxia.

Nutritional status of the animal is one factor that may influence the hepatic response to hypoxia. The literature is both sparse and contradictory on this point, with at least one report citing the protective effects of starvation (28), while others conclude that fasting exacerbates both ischemic and reperfusion injury (29-32). Despite the plethora of studies utilizing pharmacologic manipulation of hepatic xanthine oxidase activity or free radical scavengers (11-13), changes in oxidase levels have not been compared previously during hypoxia in the liver of fed and fasted animals. The mechanism(s) underlying the increased conversion to xanthine oxidase activity observed during global ischemia in the liver of fasted animals (Fig. 4) is unclear. Fasting results in a variety of changes in hepatic metabolism, including depletion of glycogen (14), decreased glutathione levels (32), and alteration of the hormonal milieu. Indeed, each of these variables has been implicated in the modulation of ischemic liver injury $(14,33,34)$, although their relation to the conversion of XDH to XOD remains undefined. The relevance of our observations, is that it now shifts the focus in organ preservation to the physiological state (e.g., nutritional status) of the organ donor.

Recently, it has been suggested that circulating elements interact with the liver $(21,35,36)$, intestine $(12,20)$, kidney (37), and heart $(3,4,18)$ during ischemia, and thereby amplify the tissue response to ischemia and/or reperfusion. Because such changes may be absent during hypoxia in ex vivo preparations, we sought to mimic a short period of in vivo ischemia using a "cardiopulmonary arrest" model. Although the imposition of a 10-min period of in vivo hypoxia had no effect on the conversion of XDH to XOD activity during subsequent ex vivo ischemia in the liver of fed rats (Fig. 4), it dramatically accelerated enzyme conversion in fasted animals (Fig. 4). This effect was evident within $30 \mathrm{~min}$ of ischemia; moreover, after only $90 \mathrm{~min}$ of ischemia, the livers of starved animals exposed to a period of cardiopulmonary arrest exhibited more edema than those of other groups, and displayed diffuse hepatocyte vacuolization that may reflect loss of volume control, as well as eosinophilic degeneration consistent with cell death (Fig. 5).

The association of marked histologic injury with the maximal increase in \% XOD activity (in the starved/CPA group) may cast doubt on the causality of increased XOD activity 
mediating subsequent reperfusion damage. There is, in fact, some evidence suggesting that the increase in xanthine oxidase is merely a reflection of cellular injury (38). Our observations, however, tend to contradict this notion. We noted significant increases in XOD conversion, unaccompanied by any detectable edema or histologic changes. More importantly, the conformationally altered XDH molecule with XOD activity (XODc or DTT-labile XOD) was increased during anoxia in the starved/CPA group (Table I). Work by McKelvey (16), as well the present data, suggests that this transformation, which is believed to reflect oxidation of several of the 14 sulfhydryl groups in the XDH molecule to disulfides (39), may precede enzymatic cleavage to the smaller XOD molecule. Thus, the increase in XOD activity is likely to be attributable to alterations in the redox state in these anoxic cells, reflecting altered cell physiology during hypoxia and not cell death with concomitant protease release.

Despite this conclusion, it is not possible, using the present model system, to resolve definitively whether the increase in $\mathrm{XOD}$ activity precedes or is a consequence of cellular injury. This is due to the fact that the sensitivity of conventional histology is suboptimal as an indicator of cell death, and it is difficult to correlate XOD activity and morphologic injury in the same cell. It is important to note, however, that the XOD theory of reperfusion injury presupposes a state of pure anoxia and high oxygen reperfusion. In fact, in most experimental systems, as well as in the corresponding pathophysiologic states in man, a state of pure anoxia does not exist. Thus, it is possible that the conversion to an increased level of xanthine oxidase during hypoxia and the related enhanced capacity to produce superoxide anion are not temporally distinct events. Hence, increased xanthine oxidase levels and free radical production (with subsequent cellular damage) may occur simultaneously during hypoxia $(16,40)$. This hypothesis would explain the cellular injury accompanying high levels of XOD observed in our cardiopulmonary arrest model, as well as the failure of many investigators to demonstrate a well-defined pattern of reperfusion injury (9, 10, Lipsky, M., and J. L. Gollan, unpublished data), despite the efficacy of allopurinol (11) and free radical scavengers (12-14) in ameliorating ischemic and so-called "reperfusion" injury.

The changes in hepatic xanthine oxidase activity and the concurrent hepatic injury are independent of white blood cells in our model system, although evidence obtained in several organ systems $(18,20,38)$, including the liver $(21)$, document endothelial cell-neutrophil interactions during the ischemia and reperfusion. Free radical modification of circulating lipids has been linked with neutrophil chemotaxis $(33,41,42)$, as well as neutrophil adherence to endothelial cells (37). Recently, this has been proposed as the mechanism underlying "reperfusion injury" and the "no-reflow" phenomenon, in which neutrophil plugging of capillaries occludes reoxygenation of hypoxic tissue after normal organ blood flow is reestablished $(4,19)$. This hypothesis and the older XOD concept are not necessarily mutually exclusive because the former assumes that free radical species may arise by the conversion to increased XOD activity and the resupply of oxygen, as described in the latter theory $(6$, $8)$. In support of this concept, allopurinol and free radical scavengers $(4,20)$ appear to effectively inhibit the occurrence of this "no-reflow" phenomenon. There are, however, major differences between the two "reperfusion injury" theories in defining the mechanistic role of xanthine oxidase. First, the site of XOD activity is shifted from the hepatocyte to the endothelial cell; in this context, it should be noted that the hepatic localization of XOD is still a topic of controversy (43-46). Second, the putative role of XOD would now be in signal transduction rather than in direct free radical-mediated injury. In emphasizing the potential effects of small amounts of free radical species generated via endothelial cell xanthine oxidase, this theory would provide an explanation for much of the previous data in the heart, which exhibits little (myocyte) XOD activity. Similarly, this hypothesis addresses concerns that the quantity of free radicals produced via XOD may be insufficient to account for major direct cellular injury (47). On the other hand, a growing literature, ascribing a role for XOD in ethanol-mediated hepatocellular injury (48), suggests that XOD may be directly involved in liver injury.

Based on these observations, it is evident that the relative importance of xanthine dehydrogenase to xanthine oxidase conversion in hypoxic or reperfusion injury still requires additional clarification, and that further advances in this area will require a detailed understanding of its physiologic control mechanisms. We have shown that the shift to xanthine oxidase activity occurs in direct relation to the extent and severity of hypoxia in functionally intact liver. Moreover, using an anoxic liver model, we have characterized two important factors, namely, the nutritional state and a period of in vivo ischemia (CPA), that markedly influence this enzymatic interconversion. The synergistic interaction of these two interventions, as well as the finding of increased DTT-labile xanthine oxidase activity, exclusively in the liver of animals subjected to fasting/ cardiopulmonary arrest, support the pathophysiologic relevance of these findings. Collectively, these data not only demonstrate a temporal course of conversion to XOD activity, consistent with recent ex vivo studies (15-17), but also document that this conversion is accelerated under well-defined conditions, to a degree consistent with the postulated role of XOD in modulating ischemic/reperfusion injury. Moreover, these findings suggest that the premorbid condition of organ donors, specifically their nutritional status and ischemic/hypoxic state, may be important factors in attaining optimal organ storage.

\section{Acknowledgments}

The experiments reported herein were conducted according to the principles set forth in the "Guide for the Care and Use of Laboratory Animals," Institute of Animal Resources, National Research Council. The authors gratefully acknowledge James M. Crawford, M.D., Ph.D., for his helpful discussion and skilled preparation of the liver photomicrographs.

This study was supported in part by National Institutes of Health grant DK-36887, and a pilot project award from the Harvard Digestive Diseases Center, National Institutes of Health grant AM-34854. Dr. Brass is a recipient of an American Liver Foundation Postdoctoral Fellowship Award.

\section{References}

1. Parks, D. A., G. B. Bulkley, and D. N. Granger. 1983. Role of oxygen free radicals in shock ischemia and organ preservation. Surgery (St. Louis). 94:428433.

2. Parks, D. A., and D. N. Granger. 1986. Contributions of ischemia and reperfusion to mucosal lesion formation. Am. J. Physiol. 250:G749-G753.

3. Schaper, J., and W. Schaper. 1983. Reperfusion of ischemic myocardium: ultra-structural and histochemical aspects. J. Am. Coll. Cardiol. 1:1037-1046. 
4. Simpson, P. J., J. K. Mickelson, and B. R. Lucchesi. 1987. Free radical scavengers in myocardial ischemia. Fed. Proc. 46:2413-2421.

5. Owens, M. L., M. L. Harrison, M. W. Wolcott, J. G. Maxwell, and J. B. Taylor. 1974. Allopurinol and hypoxanthine pretreatment of canine kidney donors. Transplantation (Baltimore). 17:424-427.

6. Roy, R. S., and J. M. McCord. 1883. Superoxide and ischemia: conversion of xanthine dehydrogenase to xanthine oxidase. In Oxy Radicals and Their Scavenger Systems. Vol. II. Cellular and Medical Aspects. R. A. Greenwald and G Cohen, editors. Elsevier/North Holland, Amsterdam. 145-153.

7. Adkison, D., M. E. Hollwarth, J. N. Benoit, D. A. Parks, J. M. McCord, and D. N. Granger. 1986. Role of free radicals in ischemia-reperfusion injury to the liver. Acta Physiol. Scand. 548(Suppl.):101-107.

8. McCord, J. M. 1985. Oxygen derived free radicals in post-ischemic tissue injury, N. Engl. J. Med. 312:159-163.

9. Jaeschke, H., C. V. Smith, and J. R. Mitchell. 1988. Reactive oxygen species during ischemia-reflow injury in isolated perfused rat liver. J. Clin. Invest. 81:1204-1206.

10. Metzger, J., and B. H. Lauterburg. 1988. Effect of allopurinol on oxidant stress and hepatic function following ischemia and reperfusion in the rat. Liver. 8:344-349.

11. Nordstrom, G., T. Seeman, and P.-O. Hasselgren. 1985. Beneficial effect of allopurinol in liver ischemia. Surgery (St. Louis). 97:679-683.

12. Atalla, S. L., L. H. Toledo-Pereyra, G. H. MacKenzie, and J. P. Cederna. 1985. Influence of oxygen-derived free radical scavengers on ischemic livers. Transplantation (Baltimore). 40:584-589.

13. Marubayashi, S., K. Dohi, K. Ochi, and T. Kawasaki. 1986. Role of free radicals on ischemic rat liver cell injury: prevention of damage by -tocopherol administration. Surgery (St. Louis). 90:184-191.

14. Jennische, E. 1984. Possible influence of glutathione on post-ischemic liver injury. Acta Pathol. Microbiol. Immunol. Scand. 92:55-64.

15. Engerson, T. D., T. G. McKelvey, D. B. Rhyne, E. B. Boggio, S. J. Snyder, and H. P. Jones. 1986. Conversion of xanthine dehydrogenase to oxidase in ischemic rat tissues. J. Clin. Invest. 79:1564-1570.

16. McKelvey, T. G., M. E. Hollwarth, D. N. Granger, T. D. Engerson, U. Landler, and H. P. Jones. 1988. Mechanisms of conversion of xanthine dehydrogenase in ischemic rat liver and kidney. Am. J. Physiol. 254:G753-G760.

17. Parks, D. A., T. K. Williams, and J. S. Beckman. 1988. Conversion of xanthine dehydrogenase to oxidase in ischemic rat intestine: a reevaluation. Am. J. Physiol. 25:G768-G774.

18. Engler, R. G., W. Schmid-Schonbein, and R. S. Pavelec. 1983. Leukocyte capillary plugging in myocardial ischemia and reperfusion in the dog. Am. J. Pathol. 11:98-111.

19. Engler, R. G., M. D. Dahlgren, D. D. Morris, M. A. Peterson, and G. W. Schmid-Schonbein. 1986. Role of leukocytes in response to acute myocardial ischemia and reflow in dogs. Am. J. Physiol. 251:H314-H322.

20. Grisham, M. B., L. A. Hernandez, and D. N. Granger. 1986. Xanthine oxidase and neutrophil infiltration in intestinal ischemia. Am. J. Physiol. 251:G567-G574.

21. Komatsu, H., A. Koo, E. Chan, M. Inoue, N. Kaplowitz, and P. H. Guth. 1989. Hepatic microvascular "no-reflow" induced by ischemia-reperfusion in the rat: role of superoxide free radicals. Gastroenterology. 96:A266. (Abstr.)

22. Schmid-Schoenbein, G. W., and R. L. Engler. 1982. Leukocyte capillary plugging in myocardial ischemia and during reperfusion in the dog. Microvasc. Res. 23:273. (Abstr.)

23. Gollan, J. L., L. Hammaker, V. Licko, and R. Schmid. 1981. Bilirubin kinetics in intact rats and isolated perfused liver. J. Clin. Invest. 67:1003-1015.

24. Van Dyke, R. W., J. L. Gollan, and B. F. Scharschmidt. 1983. Oxygen consumption by rat liver: effects of taurocholate and sulfobromophthalein transport, glucagon, and cation substitution. Am. J. Physiol. 244:G523-G531.

25. Belinsky, S. A. J. A. Popp, F. C. Kauffman, and R. G. Thurman 1984 Trypan blue uptake as a new method to study zonal hepatotoxicity in the perfused liver. J. Pharmacol. Exp. Ther. 230:755-760.

26. Waud, W. R and K. V. Rajagopalan. 1976. Purification and properties of the $\mathrm{NAD}^{+}$-dependent (type $\mathrm{D}$ ) and $\mathrm{O}_{2}$-dependent (type $\mathrm{O}$ ) forms of rat liver xanthine dehydrogenase. Arch. Biochem. Biophys. 172:354-356.
27. Barnwell, S. G., P. J. Lowe, and R. Coleman. 1983. Effect of taurochenodeoxycholate or tauroursodeoxycholate upon biliary output of phospholipids and plasma-membrane enzymes, and the extent of cell damage, in isolated perfused rat livers. Biochem. J. 216:107-111.

28. Frederiks, W. M., G. M. Fronik, F. Marx, and J. James. 1988. Influence of nutritional state in ischemic damage in the rat liver. Liver. 5:342-347.

29. Jennische, E. 1983. Effects of ischemia on hepatic cell membrane potential in the rat: differences between fed and fasted animals. Acta Physiol. Scand. 118:69-73.

30. Anundi, I., and H. DeGroot. 1989. Hypoxic liver cell death: critical $\mathrm{pO}_{2}$ and dependence of viability on glycolysis. Am. J. Physiol. 25:G58-G64.

31. Bradford, B. U., M. Marotto, J. J. LeMasters, and R. G. Thurman. 1986 Early midzonal cell death during low-flow hypoxia in the isolated perfused rat liver: protection by allopurinol. J. Pharmacol. Exp. Ther. 236:263-268.

32. Younes, M., and O. Strubelt. 1988. The involvement of reactive oxygen species in hypoxic injury to rat liver. Res. Commun. Chem. Pathol. Pharmacol. $59: 369-381$.

33. Brass, C. A., J. Paulanthony, and J. L. Gollan. 1989. Importance of nutritional state and energy source in liver preservation: hypoxic liver injury and the glucose paradox. Gastroenterology. 96:A579. (Abstr.)

34. Anundi, I., J. King, D. A. Owen, H. Schneider, J. J. Lemasters, and R. G. Thurman. 1987. Fructose prevents hypoxic cell death in liver. Am. J. Physiol. G390-G396.

35. Roll, F. J., D. M. Bissell, and H. D. Perez. 1986. Human hepatocytes metabolizing ethanol generate a non-polar chemotactic factor for human neutrophils. Biochem. Biophys. Res. Commun. 137:688-694.

36. Schlayer, H.-J. U. Karck, U. Ganter, R. Herman, and K. Decker. 1987. Enhancement of neutrophil adherence to isolated rat liver sinusoidal endothelial cells by supernatants of lipopolysaccharide-activated monocytes. Role of tumor necrosis factor. J. Hepatol. (Amst.). 5:311-321.

37. Linas, S. L., P. F. Shanley, D. Whittenburg, E. Berger, and J. E. Repine. 1988. Neutrophils accentuate ischemia-reperfusion injury in isolated perfused rat kidney. Am. J. Physiol. 255:F728-F735.

38. DeGroot, H., and A. Littauer. 1989. Hypoxia, reactive oxygen, and cell injury. Free Radical Biol. Med. 6:541-551.

39. Waud, W. R., and K. V. Rajagopalan. 1976. The mechanism of conversion of rat liver xanthine dehydrogenase from an $\mathrm{NAD}^{+}$-dependent form (type $\mathrm{D}$ ) to an $\mathrm{O}_{2}$-dependent form (type O). Arch. Biochem. Biophys. 172:365-379.

40. Marotto, M. E., R. G. Thurman, and J. J. LeMasters. 1988. Early midzonal cell death during low-flow hypoxia in the isolated, perfused rat liver. Hepatology (Baltimore). 8:585-590.

41. Petrone, W. F., D. K. English, K. Wong, and J. M. McCord. 1980. Free radicals and inflammation: the superoxide dependent activation of neutrophil chemotactic factor in plasma. Proc. Natl. Acad. Sci. USA. 77:1159-1163.

42. Fantone, J. C., and P. A. Ward. 1982. Role of oxygen-derived free radicals and metabolites in leukocyte-dependent inflammatory reactions. Am. J. Pathol. 101:397-418.

43. Ausher, C., N. Amory, C. Pasquier, and F. Delbarre. 1977. Localization of xanthine oxidase in hepatic tissue. A new histochemical method. Adv. Exp. Med. Biol. 76A:605-609.

44. Jarasch, E. D., C. Grund, G. Bruder, H. W. Heid, T. W. Keenan, and W. W. Franke. 1981. Localization of xanthine oxidase in mammary-gland epithelium and capillar endothelium. Cell. 25:67-82.

45. Parks, D., and D. N. Granger. 1986. Xanthine oxidase: biochemistry, distribution and physiology. Acta Physiol. Scand. 548(Suppl.):87-99.

46. Angermuller, A., G. Bruder, A. Volkl, H. Wesch, and H. Dariush. 1987. Localization of xanthine oxidase in crystalline cores of peroxisomes: a cytochemical and biochemical study. Eur. J. Cell Biol. 45:137-144.

47. Mitchell, J. R., C. V. Smith, H. Hughes, M. L. Lenz, H. Jaeschke, S. B. Shappell, L. H. Michael, and M. L. Entman. 1987. No evidence for reactive oxygen damage in ischemia-reflow injury. Trans. Assoc. Am. Physicians. 100:5461 .

48. Kato, S. T. Kawase, J. Alderman, N. Inatomi, and C. S. Lieber. 1990 Role of xanthine oxidase in ethanol-induced lipid peroxidation in rats. Gastroenterology. 98:203-210. 